\title{
Diffusion Poiseuille Flow of a Viscous Incompressible Binary Fluid in a Horizontal Layer with Motionless Boundaries
}

\author{
N. V. Burmasheva ${ }^{1,2, a)}$ and E. Yu. Prosviryakov ${ }^{1,2, b)}$ \\ ${ }^{1}$ Institute of Engineering Science, Ural Branch of the Russian Academy of Sciences, \\ 34 Komsomolskaya St., Ekaterinburg, 620049, Russian Federation. \\ ${ }^{2}$ Ural Federal University named after the first President of Russia B.N. Yeltsin, \\ 19 Mira St., Ekaterinburg, 620002, Russian Federation

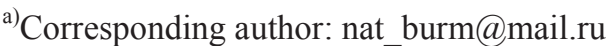 \\ b)evgen_pros@mail.ru
}

\begin{abstract}
A layered steady-state convective flow of a viscous incompressible fluid in an infinite horizontal layer induced by an inhomogeneous pressure distribution at one of the layer boundaries and by the presence of an impurity (salinity) in the fluid is considered. In addition to the equation of motion of a viscous fluid and to the law of conservation of mass for an incompressible fluid, the determining system of relations also includes an equation describing the distribution of the volume fraction of the impurity (salinity) over the entire region of the flow of the fluid under consideration. The solution of the determining system of equations is sought with the use of the class of generalized solutions, in which the velocities depend only on the vertical (transverse) coordinate, and the impurity concentration and pressure are linearly distributed along the horizontal (longitudinal) coordinates. A general solution for the determining system of equations within the chosen class is presented, and the corresponding number of boundary conditions necessary to find the values of the integration constants that appear in this general solution is formulated. A complete solution for the boundary value problem is also given. The features of the velocity field, the concentration field, and the pressure field are analyzed. The dependences of the properties of these fields on the values of parameters determining the distribution of the pressure field and the concentration field at the upper boundary of the layer are studied. It is shown that the constructed exact solution is able to describe multiple stratifications of the above-mentioned hydrodynamic. All the results obtained during the study are illustrated.
\end{abstract}

\section{BOUNDARY VALUE PROBLEM FORMULATION}

The steady-state shear flow of a viscous incompressible fluid in a plane horizontal layer of thickness $h$ is considered. To describe such flows, the following system of equations of concentration convection in the Boussinesq approximation $[1,2]$ is used:

$$
\begin{gathered}
(\boldsymbol{V} \cdot \nabla) \boldsymbol{V}=-\nabla P+v \Delta \boldsymbol{V}+g \beta C \boldsymbol{k} \\
(\boldsymbol{V} \cdot \nabla) C=d \Delta C ; \\
\nabla \cdot \boldsymbol{V}=0 .
\end{gathered}
$$

Here, $\boldsymbol{V}=\left(V_{x}(x, y, z), V_{y}(x, y, z), 0\right)$ is the velocity vector; $P(x, y, z)$ is a deviation from hydrostatic pressure divided by the average density; $C(x, y, z)$ is the concentration of the light phase of the binary mixture; $v$ and $d$ are the kinematic (molecular) viscosity of the fluid and the diffusion coefficient, respectively; $g$ is the acceleration of gravity; $\nabla$ and $\Delta$ are the Hamilton and Laplace three-dimensional operators, respectively.

The solution is sought in the class $[3,4]$ 


$$
\begin{gathered}
V_{x}=U(z) ; V_{y}=V(z) ; V_{z}=0 ; \\
P=P_{0}(z)+x P_{1}(z)+y P_{2}(z) ; C=C_{0}(z)+x C_{1}(z)+y C_{2}(z) .
\end{gathered}
$$

The class (2)-(3) describes a spatially homogeneous distribution of the velocity field and a spatially inhomogeneous distribution of the pressure field and the concentration field. Note that the physical fields in (2)-(3) act as unknowns in the system of equations (1), while the last equation of system (1) for class (2) is satisfied identically. Thus, the number of unknown functions and the number of equations in system (1) coincide.

Then, by substituting the decomposition (2)-(3) into system (1) and equating the coefficients for identical degrees of the independent variables $x$ and $y$, we find that system (1) is equivalent to the following system of ordinary differential equations:

$$
\begin{gathered}
C_{1}{ }^{\prime}=0 ; C_{2}{ }^{\prime \prime}=0 ; P_{1}{ }^{\prime}=g \beta C_{1} ; P_{2}{ }^{\prime}=g \beta C_{2} ; \\
v V^{\prime \prime}=P_{2}, v U^{\prime \prime}=P_{1}, d C_{0}{ }^{\prime \prime}=U C_{1}+V C_{2}, P_{0}{ }^{\prime}=g \beta C_{0} .
\end{gathered}
$$

The prime in system (4) denotes total derivation with respect to the variable $z$. The equations of this system are integrated sequentially, according to the order in which they are given. Note that, among the eight equations of system (4), only the first two are isolated, i.e. they are integrated independently of solutions to other equations.

During the integration of system(4), its general solution is obtained. To obtain a particular solution that corresponds to specific boundary conditions, it is necessary to determine the integration constants proceeding from these conditions.

Assume that the steady flow of a binary fluid occurs in an infinite horizontal thin layer bounded by a solid impermeable plane $z=0$, along which the fluid moves without slipping,

$$
\left.V_{x}\right|_{z=0}=\left.V_{y}\right|_{z=0}=0,\left.\frac{\partial C}{\partial \boldsymbol{n}}\right|_{z=0}=0 .
$$

Here, $\boldsymbol{n}$ is the normal to the lower boundary of the layer.

We consider the upper boundary $z=h$ to be motionless, assuming that the pressure and impurity concentration distributions are given on it as

$$
\left.V_{x}\right|_{z=h}=\left.V_{y}\right|_{z=h}=0,\left.P\right|_{z=h}=S_{0}+S_{1} x+S_{2} y,\left.C\right|_{z=h}=a x+b y .
$$

\section{EQUATION SYSTEM SOLUTION}

The solution of the system of equations (4) taking into account the boundary conditions (5)-(6) assumes the following form:

$$
\begin{gathered}
U=Z(-1+Z)\left[\psi_{1}+\psi_{2}(-2+Z)\right] ; \\
V=Z(-1+Z)\left[\gamma_{1}+\gamma_{2}(-2+Z)\right] ; \\
C_{1}=a ; C_{2}=b ; \\
C_{0}=(-1+Z)\left[\lambda_{1}\left(-1-Z-Z^{2}+Z^{3}\right)+\lambda_{2}\left(8+8 Z+8 Z^{2}-12 Z^{3}+3 Z^{4}\right)\right] ; \\
P_{1}=S_{1}+\xi_{1}(-1+Z) ; P_{2}=S_{2}+\xi_{2}(-1+Z) ; \\
P_{0}=S_{0}+\xi_{3}(-1+Z)^{2}\left(-7-4 Z-Z^{2}+2 Z^{3}\right)+\xi_{4}(-1+Z)^{2}\left(11+6 Z+Z^{2}-4 Z^{3}+Z^{4}\right) .
\end{gathered}
$$

Here, 


$$
\begin{gathered}
Z=z / h, \psi_{1}=h^{2} S_{1} /(2 v), \psi_{2}=a g \beta h^{3} /(6 v), \gamma_{1}=h^{2} S_{2} /(2 v), \gamma_{2}=b g \beta h^{3} /(6 v), \\
\lambda_{1}=\left(a S_{1}+b S_{2}\right) h^{4} /(24 d v), \lambda_{2}=\left(a^{2}+b^{2}\right) g h^{5} \beta /(360 d v), \\
\xi_{1}=a g \beta h, \xi_{2}=b g \beta h, \xi_{3}=\left(a S_{1}+b S_{2}\right) g h^{5} \beta / 240 d v, \xi_{4}=\left(a^{2}+b^{2}\right) g^{2} h^{6} \beta^{2} /(720 d v) .
\end{gathered}
$$

\section{INVESTIGATION OF THE VELOCITY FIELD}

Note that the expressions of system (7) for the velocities $U, V$ differ only in their coefficients, one of which describes the influence of the horizontal (longitudinal) pressure gradient, and the other describes the longitudinal (horizontal) concentration gradient. This means that each of the velocities $U, V$ results from two streams, namely the Poiseuille flow and the diffusion flow.

We consider the properties of the velocity $U$ and then extend the results to the behavior of the velocity $V$. Obviously, the velocity $U$ can take zero values at the boundaries of the fluid layer under study (at $Z=0$ and at $Z=1$ ), as well as inside the layer, if the equation

$$
\psi_{1}+\psi_{2}(-2+Z)=0
$$

has roots in the interval $(0,1)$. Obviously, there can be only one such root, and it is determined by the formula

$$
Z=2-\frac{\psi_{1}}{\psi_{2}}=2-\frac{3 S_{1}}{a g \beta h}
$$

This root belongs to the interval of interest $(0,1)$ only if the following inequality holds:

$$
\left(\psi_{1}-2 \psi_{2}\right)\left(\psi_{1}-\psi_{2}\right)<0 \text {. }
$$

Therefore, similarly, the velocity $V$ can have a zero point inside the layer if

$$
\left(\gamma_{1}-2 \gamma_{2}\right)\left(\gamma_{1}-\gamma_{2}\right)<0
$$

The corresponding $U, V$ velocity profiles are shown in Fig. 1.

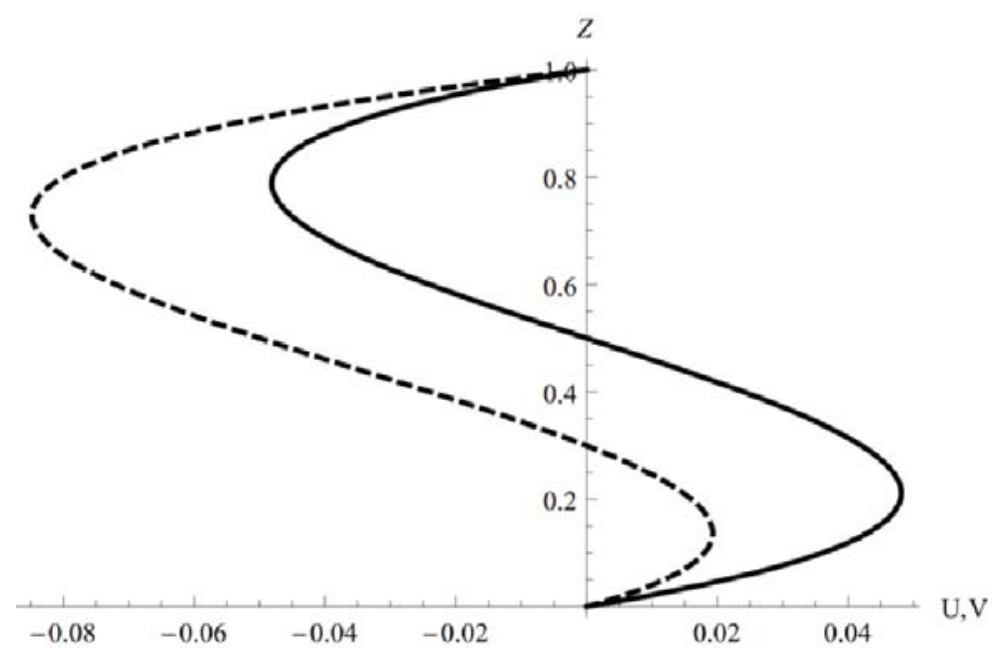

FIGURE 1. The behavior of the velocities $U$ (solid line) and $V$ (dashed line) 
The presence of zero points inside the fluid layer means the existence of regions with a reverse flow in the layer [5-10]. In order for the velocities $U, V$ to vanish at one point, the following equality must hold:

$$
\frac{S_{1}}{a}=\frac{S_{2}}{b} .
$$

This equality means that the existence of a global stagnation point in the velocity field (the point at which $\boldsymbol{V}=0$ ) is possible only if the diffusion effects are consistent with the effects caused by inhomogeneous pressure distribution.

\section{INVESTIGATION OF THE CONCENTRATION FIELD}

According to the exact solution (7), the longitudinal gradients of the concentration field prove to be constant. Therefore, the main features in the behavior of the concentration field are determined by the background concentration $C_{0}$; as the zero value of the background concentration $C_{0}$, its value at the upper boundary of the layer is taken.

The background concentration is determined by the interaction of two streams. One of them is caused by the inhomogeneous distribution of impurity at the upper boundary of the fluid layer, and the other occurs due to the cross effect of the inhomogeneous distributions of pressure and impurity. Similarly to the study conducted for the velocity field, it can be shown that background concentration can be stratified into two zones relative to the reference value (Fig. 2).

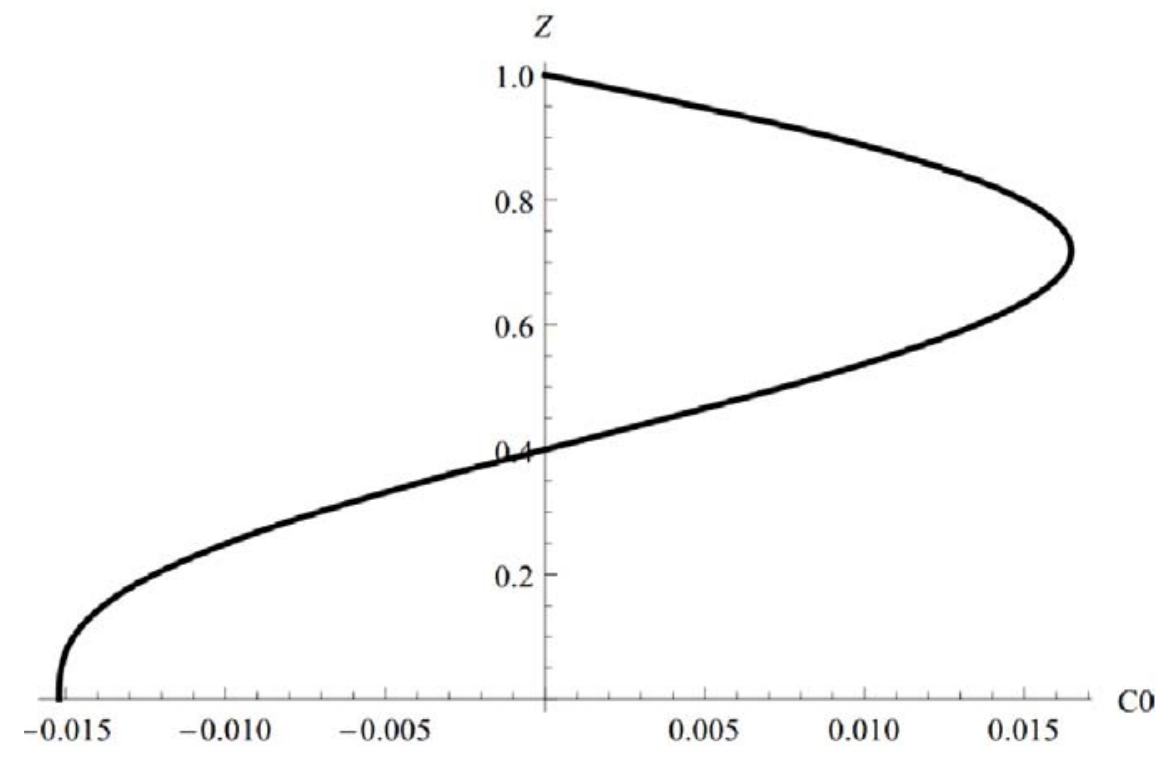

FIGURE 2. The behavior of background concentration $C_{0}$

Note that the ability of background concentration to take negative values indicates only that in the layer there can be regions where the impurity concentration is less than its value at the upper boundary.

\section{INVESTIGATION OF THE PRESSURE FIELD}

Unlike the concentration field, the longitudinal pressure gradients $P_{i}(i=1,2)$ can vanish inside the layer. The following double inequality suffices for this:

$$
S_{i}\left(S_{i}-\xi_{i}\right)<0
$$

Having analyzed the equation 


$$
P_{0}{ }^{\prime}=g \beta C_{0}
$$

of system (4), we can conclude that the number of extrema of background pressure $P_{0}$ coincides with the number of zero points of background concentration $C_{0}$. Consequently, background pressure $P_{0}$ can be stratified into three zones (Fig. 3).

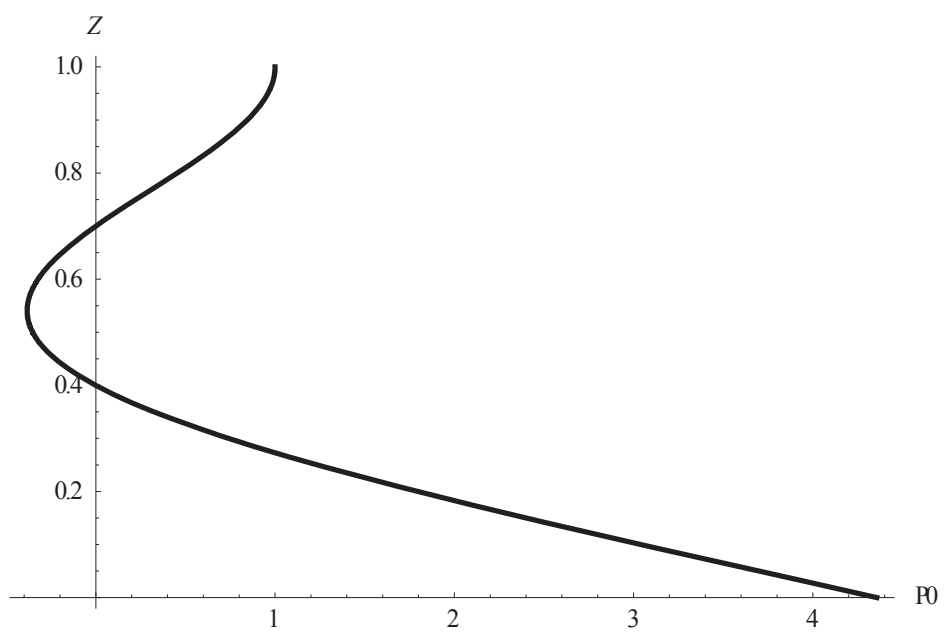

FIGURE 3. The behavior of background pressure $P_{0}$

\section{CONCLUSION}

A new solution describing the effect of the inhomogeneous distribution of the pressure field on diffusive convective flows of a viscous incompressible fluid has been proposed. It has been shown that this solution is able to describe the stratifications of hydrodynamic fields. Moreover, the number of stratification points is different for different fields.

\section{REFERENCES}

1. G. Z. Gershuni and E. M. Zhukhovitskii, Convective Stability of Incompressible Fluids (Wiley/Keter Press, Jerusalem, 1976).

2. D. D. Joseph, Stability of Fluid Motions (Springer-Verlag, Berlin, Heidelberg, New York, 1976).

3. N. V. Burmasheva and E. Yu. Prosviryakov, Vestn. Samar. Gos. Tekhn. Univ., Ser. Fiz.-Mat. Nauki 21(1), 180-196 (2017).

4. N. V. Burmasheva and E. Yu. Prosviryakov, Vestn. Samar. Gos. Tekhn. Univ., Ser. Fiz.-Mat. Nauki 21(4), 736-751 (2017).

5. V. Shtern, Counterflows. Paradoxical Fluid Mechanics Phenomena (Cambridge University Press, Cambridge 2012).

6. J. M. Dorrepaal, Journal of Fluid Mechanics 163(1), 141-147 (1986).

7. J. T. Stuart, Journal of the Aerospace Sciences 26(2), 124-125 (1959).

8. P. Riesco-Chueca and J. F. De la Mora, Journal of Fluid Mechanics 214, 639-663 (1990).

9. G. A. Ostroumov, Free convection under the condition of the internal problem (National Advisory Committee for Aeronautics, Washington, NACA Technical Memorandum 1407, 1958).

10. R. V. Birikh, J. Appl. Mech. Tech. Phys. 7(3), 43-44 (1966). 